\title{
Grouping Algorithms for Informative Array Testing in Disease Surveillance
}

\author{
David Sokolov \\ ds0016@mix.wvu.edu
}

Follow this and additional works at: https://researchrepository.wvu.edu/etd

Part of the Disease Modeling Commons, and the Other Applied Mathematics Commons

\section{Recommended Citation}

Sokolov, David, "Grouping Algorithms for Informative Array Testing in Disease Surveillance" (2021). Graduate Theses, Dissertations, and Problem Reports. 8305.

https://researchrepository.wvu.edu/etd/8305

This Thesis is protected by copyright and/or related rights. It has been brought to you by the The Research Repository @WVU with permission from the rights-holder(s). You are free to use this Thesis in any way that is permitted by the copyright and related rights legislation that applies to your use. For other uses you must obtain permission from the rights-holder(s) directly, unless additional rights are indicated by a Creative Commons license in the record and/ or on the work itself. This Thesis has been accepted for inclusion in WVU Graduate Theses, Dissertations, and Problem Reports collection by an authorized administrator of The Research Repository @ WVU. For more information, please contact researchrepository@mail.wvu.edu. 
Graduate Theses, Dissertations, and Problem Reports

2021

Grouping Algorithms for Informative Array Testing in Disease Surveillance

David Sokolov

Follow this and additional works at: https://researchrepository.wvu.edu/etd

Part of the Disease Modeling Commons, and the Other Applied Mathematics Commons 


\title{
Grouping Algorithms for Informative Array Testing in Disease Surveillance
}

\author{
David Sokolov \\ Thesis submitted \\ to the Eberly College of Arts and Science \\ at West Virginia University \\ in partial fulfillment of the requirements for the degree of \\ Master of Science in \\ Applied Mathematics \\ Casian Pantea, Ph.D., Chair \\ Brian Hendricks, Ph.D. \\ James Broda, Ph.D. \\ Department of Mathematics
}

Morgantown, West Virginia

2021

Keywords: group testing, pooled testing, COVID-19, informative array testing, disease surveillance

Copyright 2021 David Sokolov 


\title{
ABSTRACT \\ Grouping Algorithms for Informative Array Testing in Disease Surveillance
}

\author{
David Sokolov
}

In order to maintain normal operations and prevent unnecessary morbidity and mortality during times of disease outbreak, institutions find a need to conduct frequent and widespread testing of their constituents, often under significantly limited testing resource constraints. Faced with the challenge of how best to allocate these limited resources to maximum effect, institutions are increasingly turning to group (or "pooled") testing, which involves testing strategically-chosen groups of patient samples rather than individual samples, producing significant testing resource savings under certain regimes of disease prevalence. While group testing can be conducted without any a priori knowledge of individual disease risk probabilities, incorporating such knowledge - a process called informative group testing - to assign testing groups has the potential to further enhance testing efficiency. Here, we focus on one particular informative group testing procedure which groups samples into two-dimensional arrays for disease status identification (so-called informative array testing). While others have reported algorithms to optimize the construction of a testing array given its constituent samples, we focus instead on algorithms to assign array groups from a population with heterogenous disease risks. We propose two new array assignment strategies - concentrated risk and dispersed risk array assignment - and compare their performance to random array assignment by simulating informative array testing on a heterogenous-risk population. Overall, our results suggest that informative array testing is surprisingly agnostic to array assignment strategy, with one potential exception at high disease prevalence. Furthermore, our consideration of two distinct performance metrics reveals nuance in choosing an optimal informative array testing strategy with regards to both test savings and case identification efficiency. 


\section{Contents}

Table of Contents

List of Figures

List of Tables

Acknowledgments vi

1 Infectious Disease Surveillance as a Resource Allocation

$\begin{array}{ll}\text { Problem } & 1\end{array}$

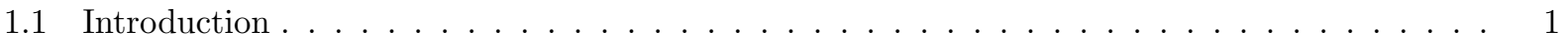

1.2 Uninformative ("Naïve") Group Testing . . . . . . . . . . . . . . . . . . . . 2

1.3 Informative Group Testing . . . . . . . . . . . . . . . . . . . . 3

1.4 Common Informative Group Testing Techniques _ . . . . . . . . . . . . . . . . . 4

1.4.1 Informative Dorfman and Hierarchical Testing . . . . . . . . . . . . . . . . . 4

1.4 .2 Informative Array Testing . . . . . . . . . . . . . . . . . . . 4

1.5 Project Aims and Scope $\ldots \ldots \ldots \ldots \ldots \ldots$

2 Investigating Grouping Algorithms for Informative Array

$\begin{array}{ll}\text { Testing } & 7\end{array}$

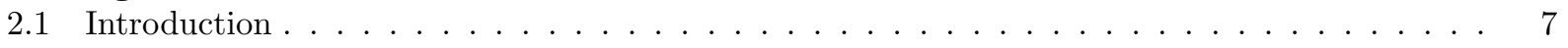

2.2 Population Construction and Disease Risk Assignment . . . . . . . . . . . . . . 7

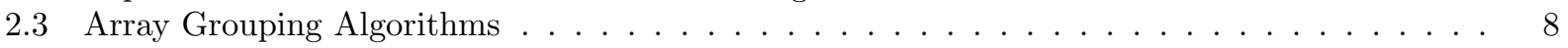

2.3.1 Random Array Assignment . . . . . . . . . . . . . . . . . . . . . 8

2.3.2 "Concentrated Risk" Array Assignment . . . . . . . . . . . . . . . . . . . . 8

2.3.3 "Dispersed Risk" Array Assignment . . . . . . . . . . . . . . . . . . . . . . . . 8

2.4 Simulation Workflow . . . . . . . . . . . . . . . . . . . . . . 9

2.4.1 Key Simplifying Assumptions . . . . . . . . . . . . . . . . . . . . . . . 9

2.4 .2 Simulation Steps . . . . . . . . . . . . . . . . . . . . . . . . . 10



3 Results $\quad 11$

3.1 Array testing simulation recapitulates key group testing phenomena . . . . . . . . . . 11

3.2 Testing and decoding efficiencies appear largely agnostic to array assignment strategy $\ldots \ldots 11$

4 Conclusions and Future Work 13

4.1 Prioritizing array construction over assignment in optimizing informative array testing . . . 13

4.2 The importance of balancing testing and decoding efficiency when considering informative array testing strategies . . . . . . . . . . . . . . . . . . 14

4.3 Future Work . . . . . . . . . . . . . . . . . . . . . . . . 14

$\begin{array}{ll}\text { References } & 14\end{array}$

5 Appendix $\quad 17$

5.1 Supplemental Figures $\ldots \ldots \ldots \ldots \ldots \ldots \ldots \ldots \ldots$

5.2 R Code . . . . . . . . . . . . . . . . . . . . . . . . . . . . 17 


\section{List of Figures}

1 Informative array construction schemes $\ldots \ldots \ldots \ldots \ldots \ldots$

2 Array assignment schemes . . . . . . . . . . . . . . . . . . 8

3 Testing simulation workflow $\ldots \ldots \ldots \ldots \ldots \ldots \ldots \ldots$

4 Testing efficiency of array assignment algorithms across disease prevalence and array size . . 12

5 Decoding efficiency of array assignment algorithms across disease prevalence and array size $\quad 12$

6 Testing efficiency of array assignment algorithms across disease prevalence and array size;

gradient and spiral array construction methods . . . . . . . . . . . . . 17 


\section{List of Tables}

$1 \quad$ Decoding efficiencies across prevalence and array size $\ldots \ldots \ldots \ldots \ldots \ldots$ 


\section{Acknowledgements}

I'd like to thank my family and friends, whose unwavering love and support astonishes me every day, my advisor, Casian, for sticking with me through hard times, and the many bright, inspiring, thoughtful, and talented people who made my time at WVU worth every second. 


\section{Infectious Disease Surveillance as a Resource Allocation Problem}

\section{$1.1 \quad$ Introduction}

Communicable disease is a ubiquitous and ever-evolving feature of the human species. While organized societies have long realized a need to effectively monitor and control infectious disease, today's globalized world is one in which disease outbreaks have the potential to spread extremely rapidly and cause significant global disruptions if left unchecked. Our technological capabilities to detect, diagnose, and treat disease have never been stronger - still, the ongoing COVID-19 pandemic has revealed the inherent difficulties of managing a novel pathogen which emerges unexpectedly and spreads throughout the globe in a matter of months $[9]$.

As COVID-19 vaccines become more universal and population susceptibility correspondingly decreases, institutions on all levels - governments, businesses, schools, and other administrative entities - realize a need for effective disease surveillance in order to maintain normal operations and prevent unnecessary morbidity and mortality. Current views in the field agree that effective disease mitigation and containment depends on frequent and massive testing of susceptible populations [18], with one study even estimating that colleges should screen the entire student population every 2 days in order to prevent further outbreaks [15]. Considering that the COVID-19 pandemic disrupted global supply chains across the board [20] including those necessary to manufacture testing reagents [1], the question of disease surveillance quickly becomes a question of resource allocation - how can institutions, who need to control disease outbreaks among their constituents, make best use of necessarily limited testing resources?

With specific regards to disease surveillance, group testing — also known as pooled testing — has emerged as a viable method to conserve resources by extending the predictive power of a single diagnostic test. First reported by Dorfman in 1943 as a means of screening army recruits for syphilis [5], group testing takes advantage of many diagnostic tests' abilities to be multiplexed; that is, for one test to be used on a mixture of multiple patient samples. In a typical group testing setup, multiple patient samples will be combined and tested with a single diagnostic test. If the test returns a negative result, the entire group is presumed negative, while a positive test result necessitates the need for further testing on that group (sometimes known as "retesting" or "decoding") to definitively identify the patients responsible for the positive result [4]. Group testing has the potential to significantly reduce testing resource expenditure under certain conditions; indeed, 
a group of size $n$ which tests negative effectively saves $n-1$ tests (which would otherwise be used to test each patient individually). Intuitively, the relative test savings of group testing increases with decreasing disease prevalence in the population [4]. By making better use of fewer testing resources and shortening the time required to screen large populations for disease, group testing is increasingly recognized as effective and necessary to control present outbreaks and prevent future ones [3].

\subsection{Uninformative ("Naïve") Group Testing}

Unsurprisingly, diverse algorithms exist for both major stages of the group testing approach (initial group formation/testing and subsequent decoding of positive groups). While these algorithms vary in their relative test-saving efficiencies, robustness to disease and test parameters, and implementation complexity, they can be broadly classified into two groups: those which are agnostic to heterogeneity in individual disease risk (uninformative or "naïve" group testing algorithms) and those which consider patient-to-patient variation in disease risk to further optimize test savings (informative group testing algorithms). While uninformative group testing approaches may consider population-level estimates of disease prevalence to optimize group sizes and can offer significant savings over individual testing [7], they ultimately treat each patient sample as identical with regards to group assignment and subsequent decoding. What follows is a brief discussion of several common uninformative group testing approaches.

The most straightforward uninformative group testing approach is known as Dorfman testing after its original proposer. In this scheme, the testing population is randomly assigned to groups of equal size, each of which are tested using a single test - groups which test positive are subsequently decoded using individual testing [5]. One step further than this are the so-called "hierarchical" group testing schemes (sometimes known as "multistage" or "splitting" algorithms). In these approaches, initial group assignment is similar to Dorfman testing, but positive groups are split into smaller groups, which are again tested. Positive groups can either be split in half ("binary splitting"), or into multiple smaller pools of different sizes, optimized according to disease prevalence. This sequential group splitting can be repeated as many times as desired, but in practice algorithms with more than four splitting stages are disfavored as the testing is too time-consuming [4].

All of the aforementioned tests are sometimes classified under the umbrella term "adaptive" or "sequential" testing schemes - once initial groups are formed, they are sequentially retested and refined to decode positive individuals. The adaptiveness of these approaches stems from the ability to optimize group size at each step based on disease prevalence and the results of previous testing stages, and multiple such algorithms 
have been developed over the past several years [4]. In contrast, so-called "non-adaptive" testing schemes take a different approach - each individual sample is first assigned to multiple distinct groups, all groups are tested, and combinatorial approaches are used to decode positive individuals after this single round (or sometimes one additional round) of testing.

One of the most popular non-adaptive group testing approaches is matrix, or array, testing [13]. The premise of this approach is to form individual samples in a two-dimensional array, then group test each row and column of that array. Samples occurring at the intersection of a positive row and column are retested to decode their disease status, yielding complete knowledge of a population's disease status after only two rounds of testing. With small modifications to account for imperfect tests, various array testing methodologies have been shown to regularly outperform Dorfman testing and see application in many workflows for population disease surveillance $[4,11,16]$. A major benefit of the array testing approach is the reduced testing time associated with fewer testing rounds - in situations where adequate disease surveillance and contact tracing depends on rapid case identification, non-adaptive approaches like array testing allow institutions to be more responsive to changing infection landscapes than if they had relied on slower, multistage sequential testing approaches [11].

Beyond array testing, more complex non-adaptive group testing algorithms exist which rely on strategic sample distribution among multiple pools and subsequent case identification using sophisticated decoding algorithms. While these approaches are beyond the scope of this investigation, they promise further efficiency improvements over array testing, and many are currently undergoing clinical validation [4]. One potential downside of these approaches is their relative complexity - some worry that real-world implementation of these strategies (which require complicated sample pooling and dedicated decoding algorithms) in testing laboratories carry increased risks of human error and case misidentification.

\subsection{Informative Group Testing}

In contrast to uninformative approaches, informative group testing algorithms take a major step forward in considering individual disease risk heterogeneity when optimizing testing groups. Collectively, these approaches seek to leverage available data - clinical, epidemiological, or otherwise - to predict each individual's probability of testing positive, and use these predictions to construct groups in such a way as to maximize test savings. While informative testing strategies depend on the availability and reliability of patient risk data, they demonstrate significant performance improvements over their uninformative counterparts with oftentimes negligible losses in screening accuracy $[13,12]$. Of particular importance to containing disease 
epidemics, informative group testing strategies show greater robustness to disease prevalence than uninformative methods - by strategically separating high- and low-risk patient samples, informative testing methods often remain effective at higher disease prevalence, where traditional group testing methods falter [6].

\subsection{Common Informative Group Testing Techniques}

\subsubsection{Informative Dorfman and Hierarchical Testing}

From a big-picture perspective, informative Dorfman and hierarchical schemes seek to minimize test usage by maximizing the occurrence of negative-testing groups. Two specific strategies described by McMahan and colleagues are the so-called Threshold Optimal Dorfman (TOD) and Pool-Specific Optimal Dorfman (PSOD) algorithms, which both seek to minimize the expected number of tests utilizing greedy algorithms and realistic constraints [12]. The TOD algorithm works by individually testing all samples above an optimal upper risk threshold and partitioning all lower-risk samples into equally sized groups of optimal size $n$ determined empirically. The PSOD approach rejects the idea of a common group size in favor of optimally-computed groups of various sizes, dictated by the disease risk landscape of the population in question. Heuristically, one can understand this algorithm as favoring larger groups of low-risk individuals and concentrating high-risk individuals into fewer, smaller groups.

An alternative to the two algorithms above was recently proposed by Malinovsky and colleagues [10]. Their approach begins by partitioning a population into variable-sized groups and testing those groups. Positive groups are further subdivided and tested-if, for instance, group $A$ is split into subgroups $A_{1}$ and $A_{2}$ and $A_{1}$ returns a negative test, $A_{2}$ is then inferred to contain the positive case, and is further subdivided and tested. This process is repeated until all samples are decoded, and an optimal testing configuration (OTC) (i.e. an optimal collection of groupings) is obtained by a backwards induction process which first finds the OTC for two samples, then three, etc. While the principals behind this approach and PSOD are similar, this algorithm claims several useful advantages over PSOD (for more details, see [10]), including a faster computation time.

\subsubsection{Informative Array Testing}

As is usually the case for informative group testing procedures, informative array testing represents a slight modification of traditional (uninformative) array testing to account for the extra knowledge of individual patient disease risks. Unlike hierarchical testing approaches, an informative array approach presents two 
distinct steps at which to incorporate individual disease risk probabilities: partitioning of populations into array groups, and construction of individual arrays from these groups.

Towards the latter step, two specific square array designs have been proposed; namely, "gradient" and "spiral" array construction algorithms [13]. Both of these seek to minimize the number of positive array rows and columns by grouping high-risk samples in close proximity to each other. Gradient construction accomplishes this by placing the highest-risk sample in the first slot of the array, and sequentially filling columns downward in order of decreasing risk (Figure 1). Meanwhile, spiral construction starts identically to gradient, but then constructs concentric rings ordered by decreasing risk (Figure 1). Simulation studies confirm that both spiral and gradient constructions outperformed random array constructions across a range of disease prevalence, with gradient performing slightly more efficiently than spiral design [13]. Furthermore, at prevelances below 10\%, gradient array testing also outperformed the PSOD algorithm, though it was not as efficient as Sterrett testing, a hierarchical testing strategy which has fallen out of favor due to its potentially large amounts of sequential testing stages [2].

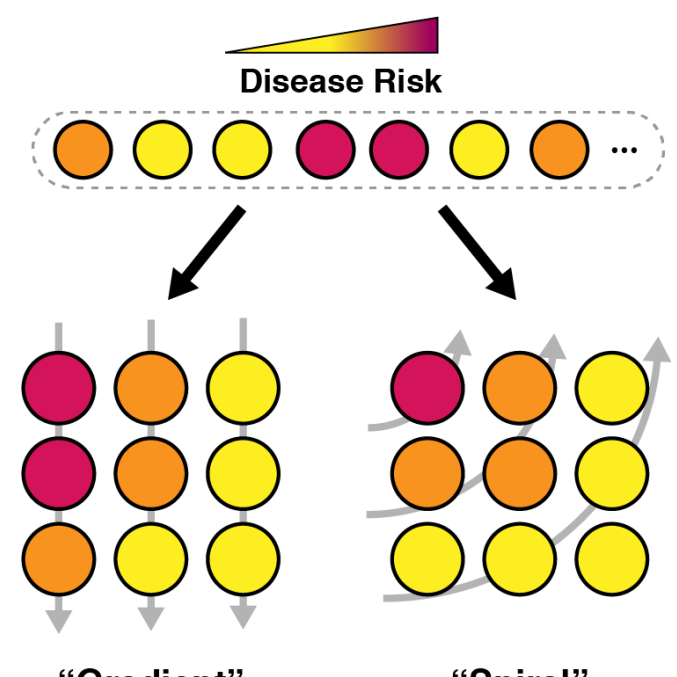

"Gradient" "Spiral"

Figure 1: Schematic depicting two optimal array construction strategies (Gradient and Spiral Construction) given individual disease risk probabilities. Circles represent individuals, colored according to disease risk. Grey arrows illustrate the array filling order by descending risk.

One other recent study describes an improved approach to informative array testing, slightly generalizing the concept of array testing to construct a series of groups, any two of which have only a single sample in common [19]. While these authors show efficiency gains using their algorithm, their approach raises potential concerns about ease of implementation and falls beyond the scope of this project. 


\subsection{Project Aims and Scope}

The aim of the current project follows naturally from a surprising lack of results concerning the first step of informative array testing described above - namely, how best to partition a population into array groups according to individual disease risk probabilities. Stated differently, once a group of samples with known risks is collected, multiple algorithms exist to strategically construct an array with a minimum expected number of positive rows and columns; however, given a population with known risks, the method of assigning individual samples to arrays in the first place has - to our knowledge - not been studied, and was hypothesized to affect the overall efficiency of the array testing algorithm.

We hypothesized that this partitioning of population into array groups is not trivial, and would in fact differ from similar grouping algorithms employed in hierarchical methods such as PSOD. This hypothesis stemmed from the supposition that testing arrays tend to perform better with fewer positives in them (for example, a single positive sample in an array can be pinpointed after only one round of testing, assuming perfect tests), while hierarchical grouping optimization often seeks to concentrate positive samples to relatively few groups [4]. Along these lines, we also must consider that crude test savings are not the only useful performance metric to evaluate group testing algorithms - for instance, the percentage of the population which are completely decoded after one round of testing is relevant to institutions conducting frequent disease surveillance, and differ drastically between optimal array and non-array testing strategies [11].

Thus, this project aims to investigate informative array testing - specifically, the methods by which a population is partitioned into array groups using disease risk probabilities. We propose two new array grouping algorithms and compare their performance to random array assignment. In addition, we compare our array grouping algorithms to individual testing on a simulated population across a range of disease prevalence. Overall, this project comprises an initial foray into further optimizing informative array testing for disease, and serves as a foundation for further research improving upon model parameters and - eventually — clinical investigations to investigate the effectiveness of informative array testing in practice. 


\section{Investigating Grouping Algorithms for Informative Array Testing}

\section{$2.1 \quad$ Introduction}

To investigate optimal methods of partitioning a population into array groups for informative array testing, we use random assignment as a baseline (and the apparent current standard in the field). In search of more efficient array assignment algorithms, we consider two conceptual strategies: the first aims to maximize the number of negative arrays (that is, arrays with no positive individuals) by concentrating the highestrisk individuals in relatively few arrays, while the second aims to maximize the number of arrays with few positives by systematically dispersing the highest-risk individuals among the arrays. Accordingly, we refer to these two strategies as "concentrated risk" and "dispersed risk" algorithms. Once array groups are assigned, arrays are constructed using the gradient and spiral designs noted previously [13].

\subsection{Population Construction and Disease Risk Assignment}

A necessary first step in evaluating the performance of our group testing strategies is the simulation of a population and corresponding disease risk probabilities. To this end, we create a population of 5,000 individuals (representing, perhaps, a medium-sized U.S. college seeking to screen a portion of its student body on a given day). Each individual disease risk probability $p$ is then randomly drawn from a beta distribution with a specified mean risk value and standard deviation, as is standard in the field [13]. Recall that, given a mean risk probability $\mu$ and standard deviation $\sigma$, the corresponding beta distribution has shape parameters

$$
\alpha=\left(\frac{(1-\mu)}{\sigma^{2}}-\frac{1}{\mu}\right) \mu^{2} \quad \beta=\alpha\left(\frac{1}{\mu}-1\right)
$$

In our simulation, we assign risk probabilities from a beta distribution with $\mu$ equal to the desired average disease prevalence and a standard deviation of $\sigma=\frac{\mu}{2}$. Next, we simulate infection of the population by assuming that our disease risk probabilities are exactly accurate - that is, each risk probability represents the probability of infection for the corresponding individual. Thus, each individual is assigned a disease status ( $0=$ uninfected, $1=$ infected $)$ according to a Bernoulli random variable with parameter $p$. This produces a population with a unimodal distribution of risk probabilities and corresponding infection statuses, which we proceed to decode using our group testing algorithms. 


\subsection{Array Grouping Algorithms}

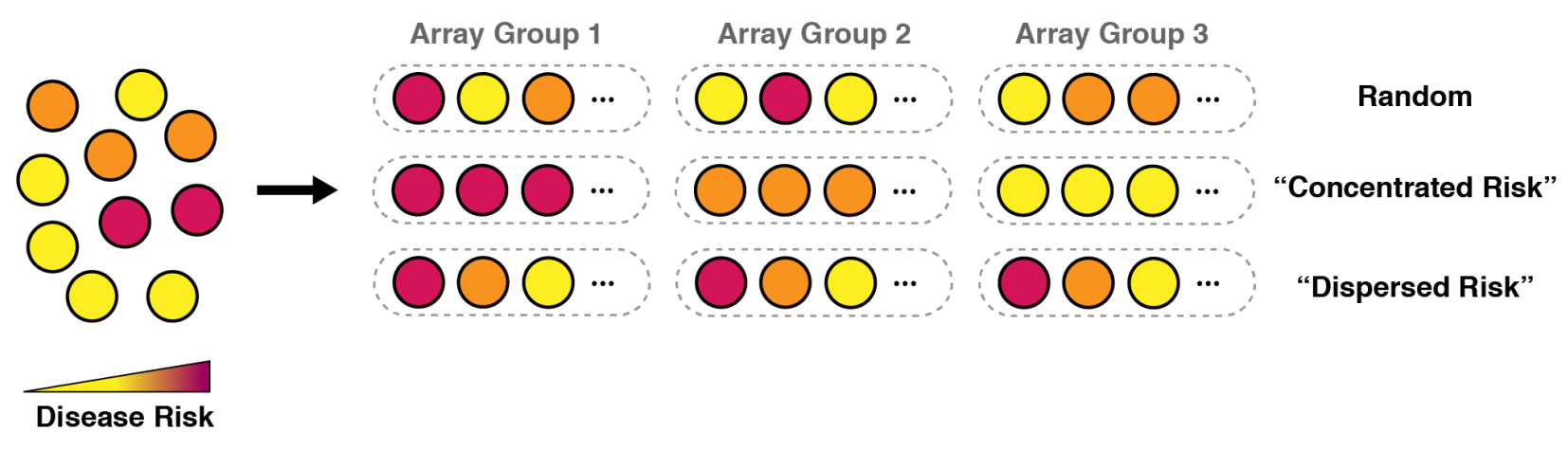

Figure 2: Schematics depicting the three array assignment schemes (Random, Concentrated Risk, and Dispersed Risk) considered in this paper. Circles represent individuals, colored according to disease risk, and dotted lines represent groups of individuals to be put into arrays for testing.

\subsubsection{Random Array Assignment}

As a baseline for comparison, we employ a random array assignment algorithm: given an array size $a$ and a population size $n$, the highest-risk $n \bmod a$ individuals are set aside for individual testing, while the remaining individuals are equally and randomly partitioned into array groups of size $a$ (Figure 2).

\subsection{2 "Concentrated Risk" Array Assignment}

As mentioned above, this algorithm seeks to maximize the number of negative arrays by concentrating the highest-risk individuals in as few array groups are possible. Given an array size $a$ and a population size $n$, the highest-risk $n \bmod a$ individuals are set aside for individual testing. From the remaining individuals, the highest-risk $a$ are grouped into an array, the next highest-risk $a$ are grouped into the next array, and so on, until all array groups are filled (Figure 2).

\subsection{3 "Dispersed Risk" Array Assignment}

This algorithm seeks to maximize the number of few-positive arrays by systematically spreading the highestrisk individuals among available array groups. Given an array size $a$ and a population size $n$, the highest-risk $n \bmod a$ individuals are set aside for individual testing. From the remaining individuals, the highest-risk individual is placed into the first array group, the second highest-risk is placed into the next array group, and so on, until all array groups are filled (Figure 2). 


\subsection{Simulation Workflow}

\subsubsection{Key Simplifying Assumptions}

While some of these assumptions are mentioned above, below we list all of the major simplifying assumptions used in our simulations, with a rationale for each:

Assumption 1: For the purposes of this initial investigation, we assume perfect tests. That is, given a positive sample, any test will return a positive result with probability 1 (i.e. test sensitivity $=1$ ), and given a negative sample, any test will return a negative result with probability 1 (i.e. test specificity $=1$ ). While no real-world diagnostic test attains $100 \%$ accuracy, imperfect tests somewhat complicate array test interpretation, and with PCR-based COVID-19 tests approaching 80\% sensitivity and 99\% specificity and recent results which indicate that testing sensitivity is secondary to other testing parameters for effective surveillance [8], we argue that this assumption is not particularly detrimental to our goals.

Assumption 2: For the purposes of this initial investigation, we assume square testing arrays of side length 3 to 8 (array size 9 to 64 ). While considering square arrays slightly simplifies analysis, our methods can easily be adapted to rectangular arrays; however, we argue that square arrays are easier to implement in practice and thus might be preferable to rectangular arrays. A maximum array size of 64 was chosen based on studies reporting unaffected COVID-19 PCR test accuracy for 8-sample pools [21, 14] and to accommodate the standard 8x12 96-well plate commonly used in testing laboratories.

Assumption 3: In conducting an array test, we adopt the standard procedure: given an array, we group each row and each column (if the array is $n$ by $n$, this produces $2 n$ groups). Each group is then tested, and samples at the intersection of a positive row and column group are then retested individually to decode their disease statuses. Importantly, we include a modification to this procedure; since we assume perfect tests, we also assume that, given only a single row and column test positive in an array, the sample at their intersection is declared positive without a second testing round. Even under a more realistic scenario where retesting these samples to confirm disease status is desired, this retesting can occur at the same time as contact tracing and quarantine measures are initiated, without losing the institution any valuable response time.

Assumption 4: As mentioned above, we assume that individual risk probabilities are exactly accurate. 
While this assumption is almost certainly unrealistic, considerations of the accuracy of risk data for informative group testing - an eminent problem in the field - are beyond the scope of the current investigation.

\subsubsection{Simulation Steps}

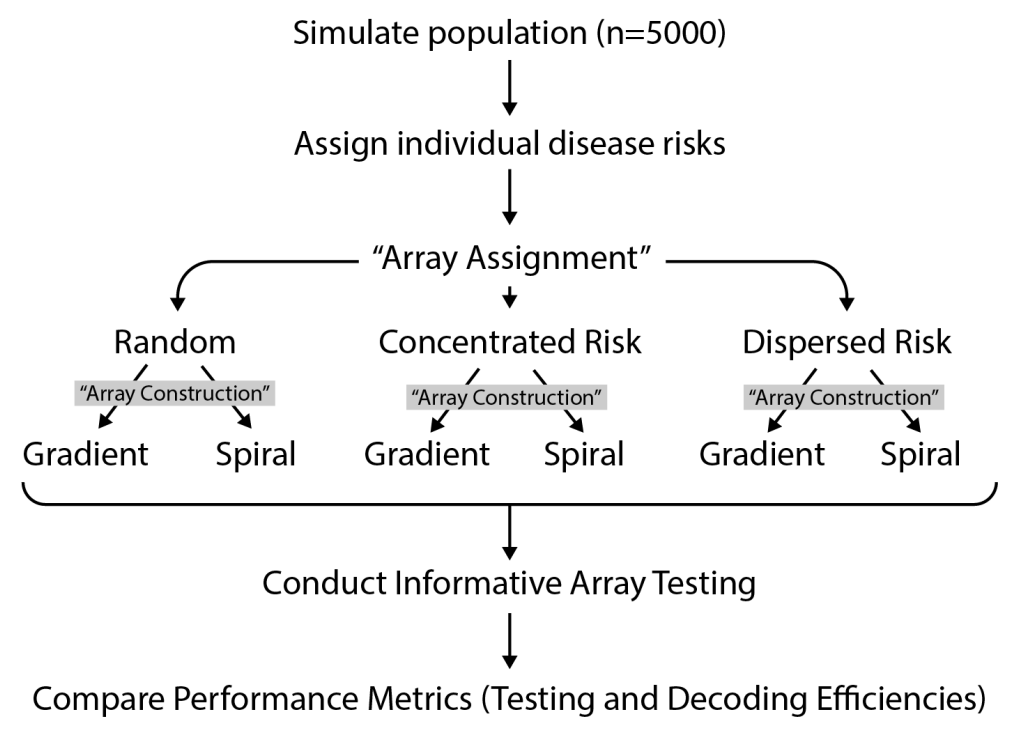

Figure 3: Schematic representing the workflow used to simulate informative array testing of a moderatelysized $(\mathrm{n}=5000)$ diseased population. Ten simulations were run and averaged for each combination of disease prevalence, array assignment method, and array construction method.

\subsubsection{Testing Performance Metrics}

In evaluating the absolute and relative performances of our group testing algorithms, we consider two key metrics. The first, testing efficiency $\left(E_{T}\right)$ is defined as the number of tests required $(t)$ as a proportion of the population $(n)$ :

$$
E_{T}=\frac{t}{n}
$$

and is standard in the field. In addition, we define another metric, decoding efficiency $\left(E_{D}\right)$ as the proportion of the population whose disease status is decoded after the first round of testing (recall that informative array testing can require up to 2 rounds of testing, and hierarchical schemes may require more). We argue that this metric is useful to institutions conducting frequent surveillance testing of large populations-in some cases, a group testing strategy which saves slightly less tests but effectively decodes a majority of the population after only a single round of testing may be more useful at facilitating rapid contact tracing and outbreak containment. 


\section{Results}

\subsection{Array testing simulation recapitulates key group testing phenomena}

As an initial observation, we note that, among all population and testing parameters, group testing efficiency remains below 1 at disease prevalence below approximately 30\% (Figure 4). This is to say that group testing uses a greater number of tests than individual testing if disease prevalence is higher than approximately $30 \%$, a threshold consistent with group testing literature [17]. In addition, larger array sizes tend to produce greater test savings (recall that, given our definition of testing efficiency, a lower magnitude is of this metric is preferable), although this effect is more pronounced at low disease prevalence (Figure 4). As expected, overall testing efficiencies are best at low disease prevalence and worsen gradually as prevalence increases; yet, even at relatively high prevalence ( $10 \%$ ), informative array testing can potentially reduce testing loads by roughly 40\%. Finally, examining the decoding efficiencies as a whole (Figure 5) underscores the advantage of array testing over other group testing approaches: at disease prevalences below approximately 10\%, informative array testing can decode over $60 \%$ (and as high as $99 \%$ !) of the testing population after only a single round of testing. Interestingly, under our simulation conditions, smaller array sizes tend to produce more desirable decoding efficiencies despite having less desirable testing efficiencies at most prevalences (Figure 5, Table 1).

With regard to array construction, our simulation recapitulates published results [13] demonstrating that, across testing and disease parameters, gradient array construction slightly outperforms spiral construction. For this reason, we focus our analysis on the gradient array construction method.

\subsection{Testing and decoding efficiencies appear largely agnostic to array assign- ment strategy}

Under our simulation conditions, it appears that both testing and decoding efficiencies within a single array size are strikingly similar among all three array assignment algorithms investigated (Figures 4 and 5). While there are prevalence regimes where dispersed risk array assignment is marginally more efficient than random array assignment, these differences are on the order of tens of tests in a population of 5000-too small to be of practical importance. One potential exception to this observation is seen at high disease prevalence and large array sizes, where the concentrated risk array assignment method begins to outperform both random and dispersed assignment strategies at prevalences past 20\%. Although also relatively small in magnitude, this performance gain may be influenced by different simulation parameters (imperfect tests and larger 

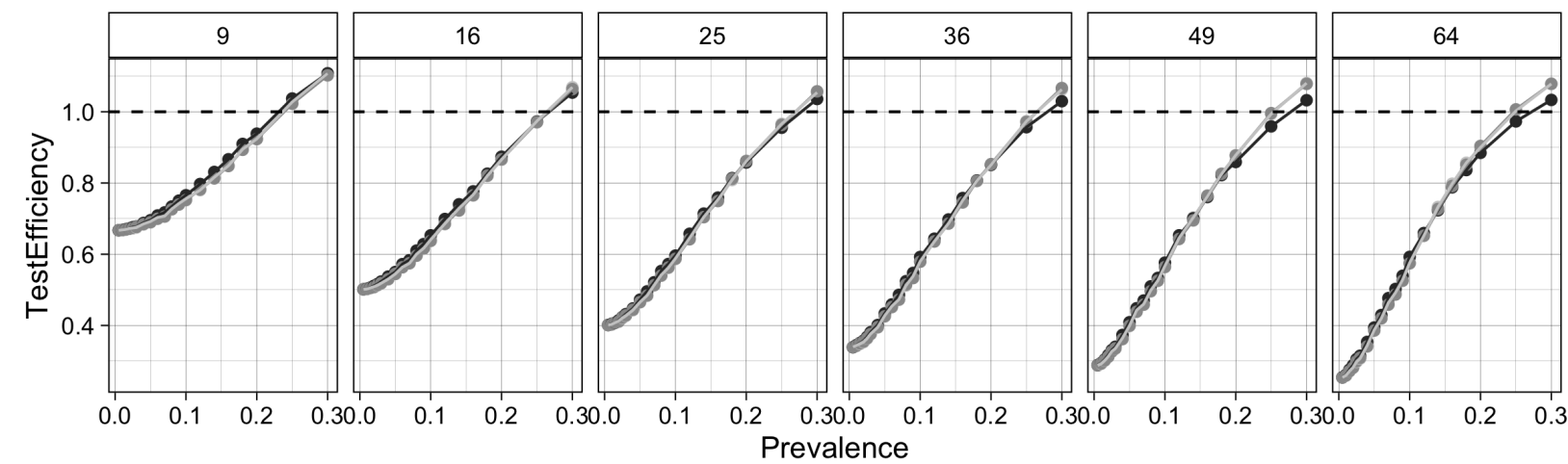

$\rightarrow$ ConcentratedGradient $\rightarrow$ DispersedGradient $\rightarrow$ RandomGradient

Figure 4: Testing efficiencies (number of tests as a proportion of the population size) of three array assignment algorithms with subsequent array construction using the gradient method. Disease prevalence ranges from $0 \%$ to $30 \%$, and panels represent differing array sizes (i.e. 36 is equivalent to 6 by 6 testing arrays). Note that the y-axis does not begin at 0 . A testing efficiency of 1 (equivalent to individual testing) is indicated with dotted lines.

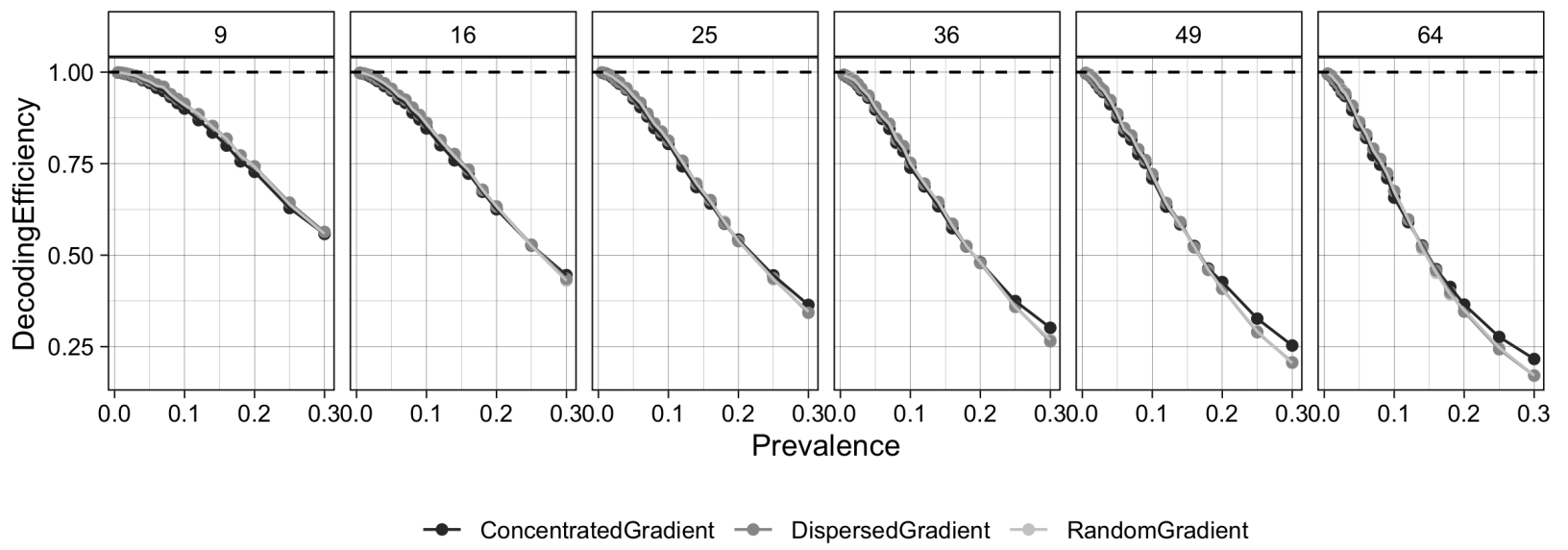

Figure 5: Decoding efficiencies (the proportion of the population whose disease status is decoded after one round of testing) of three array assignment algorithms with array construction using the gradient method. Disease prevalence ranges from $0 \%$ to $30 \%$, and panels represent differing array sizes (i.e. 36 is equivalent to 6 by 6 testing arrays). Note that the y-axis does not begin at 0 . A decoding efficiency of 1 (equivalent to decoding the entire population after one round of testing) is indicated with dotted lines.

populations), warranting further investigation. 


\begin{tabular}{|l|llllll|}
\hline & \multicolumn{7}{|c|}{ Array Size } \\
Prevalence & 9 & 16 & 25 & 36 & 49 & 64 \\
\hline 0.05 & 0.976 & 0.954 & 0.934 & 0.905 & 0.886 & 0.863 \\
0.1 & 0.913 & 0.861 & 0.813 & 0.752 & 0.721 & 0.675 \\
$0 . .2$ & 0.742 & 0.632 & 0.538 & 0.478 & 0.408 & 0.345 \\
\hline
\end{tabular}

Table 1: Decoding efficiencies (the proportion of the population whose disease status is decoded after a single round of testing) for the indicated prevalence values and array sizes using dispersed risk array assignment and gradient array construction algorithms.

\section{Conclusions and Future Work}

\subsection{Prioritizing array construction over assignment in optimizing informative array testing}

While we were unable to find published results discussing methods of array assignment in informative array testing schemes, our current simulations suggest that testing and decoding efficiency gains in informative array testing are more responsive to array construction rather than assignment. While gradient or spiral construction has been shown to outperform random array construction [13], our data suggest that any performance improvements resulting from strategic array construction are marginal. This observation may be explained in the context of our population risk landscape -in cases where individual disease risks are tightly clustered around a relatively low-risk mean, dispersing high-risk individuals and randomly dispersing all individuals may produce similar testing array groups, resulting in similar testing and decoding efficiencies.

The finding that concentrated risk array assignment slightly outperforms random and dispersed algorithms at high prevalence mimics a finding previously documented in hierarchical testing - specifically, the Threshold Optimal Dorfman (TOD) group testing algorithm was reported to perform well at high prevalences [12]. Interestingly, both TOD and concentrated risk array assignment serve to amalgamate high-risk individuals. The better performance of these algorithms at high disease prevalence may therefore suggest a more generalized group testing operating characteristic.

The possibility that informative array testing efficiency is insensitive to array assignment strategy is of potential importance to institutions seeking to conduct informative array testing for disease surveillance. As mentioned previously, effective disease surveillance is a careful balance between optimizing for testing efficiency without sacrificing implementation complexity. Notably, the ability to assign array groups randomly alleviates institutions' need to collect an entire population of samples before forming array groups-instead, laboratories may assign array groups as samples arrive in the laboratory, focusing only on constructing the 
arrays themselves, and thus reducing the possibility for human error.

\subsection{The importance of balancing testing and decoding efficiency when consid- ering informative array testing strategies}

Our simulations also suggest an interesting balance between testing and decoding efficiencies with regards to informative array testing. Specifically, we find that smaller array sizes-while generally saving fewer tests than larger arrays-tend to decode higher proportions of the population after one round of testing, especially in prevalence regimes between $10 \%$ and $20 \%$. In addition, population decoding by smaller arrays tends to be more robust to increasing disease prevalence than that of larger arrays. Decoding efficiency is potentially important for institutions requiring large-scale and nimble disease monitoring strategies; the faster cases can be identified, the quicker contact tracing protocols may be initiated to stem outbreaks [8]. Thus, our simulations reject the notion that, when it comes to informative array testing, bigger arrays are always better than smaller arrays. Institutions interested in optimizing informative array testing must carefully weigh the costs and benefits of increased tests savings and decreased decoding efficiency, and middle-of-the-road array sizes may present the optimal balance between these two considerations.

\subsection{Future Work}

Of course, the simulations presented here represent an initial foray into optimizing array assignment for informative array disease testing, and further theoretical and experimental studies are needed before definitive conclusions or recommendations can be drawn. Further development of the work described here should focus on systematically relaxing the assumptions of perfect tests and risk probabilities, and more extensive simulations of larger populations will assist in generalizing the observations noted in this report. Continued study of group testing-and informative group testing in particular-is a worthwhile venture as our capabilities to simultaneously detect disease and estimate individual disease risks continue to improve.

\section{References}

[1] M. Behnam, A. Dey, T. Gambell, and V. Talwar, COVID-19: Overcoming supply shortages for diagnostic testing, p. 8. 
[2] C. R. Bilder And J. M. TeBbs, Pooled-testing procedures for screening high volume clinical specimens in heterogeneous populations, Statistics in Medicine, 31 (2012), pp. 3261-3268.

[3] V. Brault, B. Mallein, And J.-F. Rupprecht, Group testing as a strategy for COVID-19 epidemiological monitoring and community surveillance, PLOS Computational Biology, 17 (2021), p. e1008726.

[4] E. A. Daniel, B. H. Esakialraj L, A. S, K. Muthuramalingam, R. Karunaianantham, L. P. Karunakaran, M. Nesakumar, M. Selvachithiram, S. Pattabiraman, S. Natarajan, S. P. Tripathy, And L. E. Hanna, Pooled Testing Strategies for SARS-CoV-2 diagnosis: A comprehensive review, Diagnostic Microbiology and Infectious Disease, 101 (2021), p. 115432.

[5] R. Dorfman, The Detection of Defective Members of Large Populations, The Annals of Mathematical Statistics, 14 (1943), pp. 436-440.

[6] M. Escobar, G. Jeanneret, L. Bravo-Sánchez, A. Castillo, C. Gómez, D. Valderrama, M. F. Roa, J. Martínez, J. Madrid-Wolff, M. Cepeda, M. Guevara-Suarez, O. L. Sarmiento, A. L. Medaglia, M. Forero-Shelton, M. Velasco, J. M. Pedraza-Leal, S. ReStrepo, and P. Arbelaez, Smart Pooling: AI-powered COVID-19 testing, preprint, Epidemiology, July 2020 .

[7] B. D. Hitt, C. R. Bilder, J. M. Tebbs, and C. S. McMahan, The objective function controversy for group testing: Much ado about nothing?, Statistics in Medicine, 38 (2019), pp. 4912-4923.

[8] D. B. Larremore, B. Wilder, E. Lester, S. Shehata, J. M. Burke, J. A. Hay, M. Tambe, M. J. Mina, And R. PARKer, Test sensitivity is secondary to frequency and turnaround time for COVID-19 screening, Science Advances, 7 (2021), p. eabd5393.

[9] Y.-C. Liu, R.-L. Kuo, And S.-R. Shin, COVID-19: The first documented coronavirus pandemic in history, Biomedical Journal, 43 (2020), pp. 328-333.

[10] Y. Malinovsky, G. Haber, And P. S. Albert, An optimal design for hierarchical generalized group testing, Journal of the Royal Statistical Society: Series C (Applied Statistics), 69 (2020), pp. 607-621.

[11] J. H. McDermott, D. Stoddard, P. J. Woolf, J. M. Ellingford, D. Gokhale, A. Taylor, L. A. Demain, W. G. Newman, And G. Black, A Nonadaptive Combinatorial Group Testing Strategy to Facilitate Health Care Worker Screening during the Severe Acute Respiratory Syndrome Coronavirus-2 (SARS-CoV-2) Outbreak, The Journal of Molecular Diagnostics, 23 (2021), pp. 532-540. 
[12] C. S. McMahan, J. M. Tebbs, and C. R. Bilder, Informative Dorfman Screening, Biometrics, 68 (2012), pp. 287-296.

[13] C. S. Mcmahan, J. M. Tebbs, and C. R. Bilder, Two-Dimensional Informative Array Testing, Biometrics, 68 (2012), pp. 793-804.

[14] L. Mutesa, P. Ndishimye, Y. Butera, J. Souopgui, A. Unineza, R. Rutayisire, E. L. Ndoricimpaye, E. Musoni, N. Rujeni, T. Nyatanyi, E. Ntagwabira, M. Semakula, C. Musanabaganwa, D. Nyamwasa, M. Ndashimye, E. Ujeneza, I. E. Mwikarago, C. M. Muvunyi, J. B. Mazarati, S. Nsanzimana, N. Turok, and W. Ndifon, A pooled testing strategy for identifying SARS-CoV-2 at low prevalence, Nature, 589 (2021), pp. 276-280.

[15] A. D. Paltiel, A. Zheng, and R. P. Walensky, Assessment of SARS-CoV-2 Screening Strategies to Permit the Safe Reopening of College Campuses in the United States, JAMA Network Open, 3 (2020), p. e2016818.

[16] R. M. Phatarfod And A. Sudbury, The use of a square array scheme in blood testing, Statistics in Medicine, 13 (1994), pp. 2337-2343.

[17] L. N. Theagarajan, Group Testing for COVID-19: How to Stop Worrying and Test More, arXiv:2004.06306 [q-bio, stat], (2020). arXiv: 2004.06306.

[18] R. P. Walensky and C. Del Rio, From Mitigation to Containment of the COVID-19 Pandemic: Putting the SARS-CoV-2 Genie Back in the Bottle, JAMA, 323 (2020), p. 1889.

[19] W. Xiong, J. Ding, Y. He, And Q. Li, Improved matrix pooling, Statistical Methods in Medical Research, 28 (2019), pp. 211-222.

[20] Z. Xu, A. Elomri, L. Kerbache, And A. El Omri, Impacts of COVID-19 on Global Supply Chains: Facts and Perspectives, IEEE Engineering Management Review, 48 (2020), pp. 153-166.

[21] I. Yelin, N. Aharony, E. S. Tamar, A. Argoetti, E. Messer, D. Berenbaum, E. Shafran, A. Kuzli, N. Gandali, O. Shredi, T. Hashimshony, Y. Mandel-Gutfreund, M. Halberthal, Y. Geffen, M. Szwarcwort-Cohen, and R. Kishony, Evaluation of COVID-19 RT-qPCR Test in Multi sample Pools, Clinical Infectious Diseases, 71 (2020), pp. 2073-2078. 


\section{Appendix}

\subsection{Supplemental Figures}

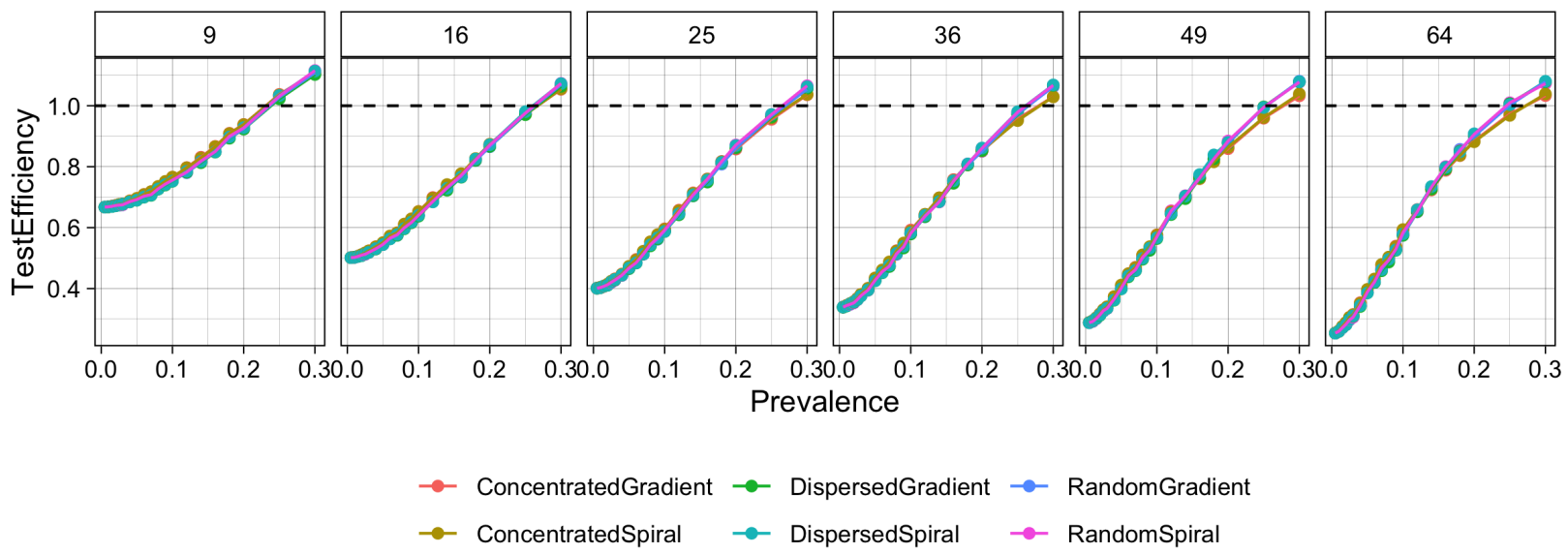

Figure 6: Testing efficiencies (number of tests as a proportion of the population size) of three array assignment algorithms with subsequent array construction using the gradient and spiral methods. Disease prevalence ranges from $0 \%$ to 30\%, and panels represent differing array sizes (i.e. 36 is equivalent to 6 by 6 testing arrays). Note that the y-axis does not begin at 0 . A testing efficiency of 1 (equivalent to individual testing) is indicated with dotted lines.

\section{$5.2 \quad \mathrm{R}$ Code}

$\mathrm{R}$ code which conducts the testing simulations described in this document and produces the source data for all figures is available at https://github.com/davemanthecaveman/Informative-Array-Testing-Thesis.git. 